\author{
Yuriy HRYSIUK ${ }^{1}$ \\ Artem LABUTA ${ }^{2}$ \\ Ruslan GRYGORENKO ${ }^{3}$
}

\title{
ЛОГИСТИЧЕСКИЙ ПОДХОД КАК ФАКТОР ЭКОНОМИЧЕСКОЙ БЕЗОПАСНОСТИ ПРЕДПРИЯТИЯ
}

\begin{abstract}
В статье рассматривается применение логистики как путь достижения экономической безопасности и стабильности предприятия. Определены понятия и виды риска как экономической категории. Предложена оптимальная форма логистического объединения, адекватная современной экономике. Логистический сервис рассмотрен в аспекте экономической безопасности предприятия.
\end{abstract}

\section{1. ВВЕДЕНИЕ}

Экономический риск на предприятии можно представить в виде блочной системы, при этом применение логистики влияет на каждый блок. Современный уровень внедрения логистики определяет новую форму объединения логистическую сеть, которая предоставляет возможность внутренней интеграции каждого блока логистической цепи. В последнее время большое внимание отводится проблеме внедрения логистики. Применение логистики рассматривается как ключевая составляющая снижения расходов на пути продвижения товаров и услуг от производителя к конечному потребителю, концептуальная основа оптимизации производственно-коммерческой деятельности, условие конкурентоспособности современного предприятия [1]. Объем решаемых вопросов формируется на уровне первичного звена экономики - предприятия.

\section{2. ОСНОВНОЙ РАЗДЕЛ}

Потребность в безопасности присуща любой системе. Как известно, хозяйственной системой на микроуровне выступает предприятие, которое служит „структурированным элементом экономики” [4]. Поэтому экономические процессы, которые происходят в государстве, прежде всего рассматриваются с точки зрения предприятия. Это касается и проблемы безопасности государства в экономической сфере, от которой зависят все другие составляющие национальной безопасности: социально-политическая, информационная, экологическая [3]. Это положение ведет к выводу о многогранности понятия экономической безопасности. Исходя из этого,

${ }^{1}$ Dr Yuriy Hrysiuk, Katedra Transportowego Prawa i Logistyki Narodowego Uniwersytetu Transportu w Kijowie, Ukraina.

${ }^{2}$ Mgr Artem Labuta, Katedra Transportowego Prawa i Logistyki Narodowego Uniwersytetu Transportu w Kijowie, Ukraina.

${ }^{3}$ Mgr Ruslan Grygorenko, Katedra Transportowego Prawa i Logistyki Narodowego Uniwersytetu Transporty w Kijowie, Ukraina. 
экономическая безопасность должна рассматриваться в зависимости от условий функционирования предприятия. В обычных условиях безопасность можно рассматривать как экономическую стойкость, которая определяется финансовым положением, конкурентоспособностью продукции либо услуги и предприятия в целом. Экономическую угрозу здесь могут представлять: активная часть основных фондов (моральный износ), новая технология (интеграция в производственный процесс), персонал (специализация и квалификация) и др. В экстремальных условиях центром внимания должны стать защитные меры против негативного влияния внешней среды, направленные на снижение потенциального хозяйственного риска. Появление хозяйственного риска обусловлено естественными и человеческими факторами. Также немаловажная роль принадлежит экономическим факторам, диапазон названий и действий которых не ограничен. Поэтому хозяйственный риск часто отождествляется с экономическим риском. Если под риском вообще понимается „возможность наступления неблагоприятного события", то под экономическим риском имеется в виду „возможность потерь вследствие случайного характера результатов принимаемых хозяйственных решений или осуществляемых действий”. Существует и более широкое трактование понятия: „вероятность того, что предприятие понесет урон или потери, если намеченное мероприятие либо управленческое решение не осуществится, а также если были допущены просчеты или ошибки при принятии управленческих решений” [3]. Экономический риск возникает под действием как внутренних, так и внешних факторов. К внутренним принадлежат нерациональная организация производства и работы, превышение нормативных материальных и трудовых расходов, проведение внепланового ремонта, выпуск бракованных изделий. Внешние причины делятся на: объективные - колебание процентных ставок, спроса, курсов валют, цен на мировом рынке и др. и субъективные, обусловленные отношениями предприятий. Субъективные факторы отображают конъюнктуру рынка исходных материалов (рынок снабжения) и рынка готовой продукции (рынок сбыта). Повышение научно-технического уровня предприятия двояко влияет на величину его риска: с одной стороны, увеличивается возможность регулирования риска, с другой - расширяется его спектр. Проявления экономического риска можно наблюдать в разных формах (рис. 1). 


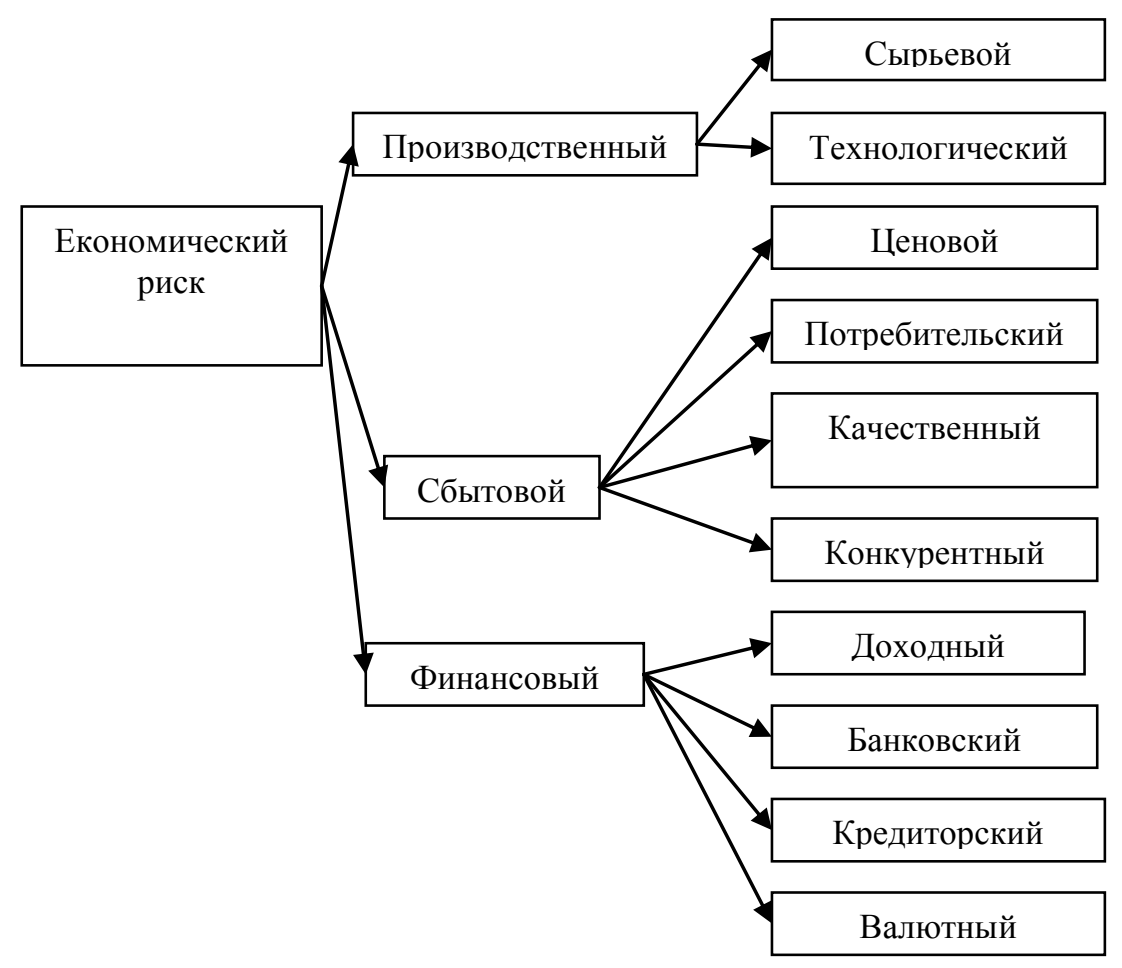

Рис. 1. Классификация видов экономического риска на микроуровне предприятия

Выделяют следующие виды и подвиды экономического риска:

1. Производственный: технологический, обусловленный отклонениями в самом процессе создания товара; сырьевой, связанный с недостаточным снабжением или снабжением некачественными материалами. 2. Сбытовой: качественный и ценовой, обусловленные несоответствием качества и цены товара с ожиданиями потребителей; потребительский - отображает изменения спроса вследствие влияния моды, уровня доходов и др.; конкурентный - предусматривает появление аналогов. 3. Финансовый: доходный, вызванный уменьшениям объема продаж и вследствие снижением доходов предприятия; кредиторский, основанный на превышении фактической кредиторской задолженности над плановой; банковский, связанный с несоответствием действий банка и предприятия, которые кредитуется им; валютный, обусловленный изменением курса национальных денежных средств (гривны) по отношению к иностранной валюте. Заметим, что производственный риск фактически влияет на все виды сбытового риска, но главное направление его действия сосредоточено на качественном и ценовом рисках. Сбытовой риск, в свою очередь, определяет так называемый прибыльный риск, т.е. риск основной деятельности предприятия. Как известно, основными направлениями снижения риска есть диверсификация, страхование, получение дополнительной информации. Фактически уменьшает риск только последний фактор, поскольку первый распределяет риск между видами продукции, а второй передает его страховой 
компании. Диверсификация, которая является расширением ассортиментов продукции и услуг, разрешает получить средний доход, снижая вероятность как максимального, так и минимального дохода. Разность между ними практически и является платой за снижение риска. В ряде случаев плата за риск имеет реальное количественное измерение: оплата страховки или стоимость приобретенной информации. В условиях внедрения логистики проблема снижения экономического риска приобретает новый характер. Фактически происходит распределение риска между всеми звеньями логистической цепи, то есть диверсификация риска. Кроме того, удлинение логистической цепи за счет включения новых звеньев естественно увеличивает объем информации внутри предприятия. Следует отметить, что объединение предприятий в логистическую цепь разрешает своевременно получать информацию, которая повышает ее стоимость. Повышение уровня информационного обеспечения приводит как к более координированной работе логистических звеньев, так и к снижению неопределенности влияния окружающей среды. Таким образом, появляется возможность планировать изменения результирующих показателей в зависимости от действия факторов макросреды и внешней микросреды. Совокупное влияние факторов внешней среды на результат деятельности предприятия (как результат можно рассматривать выручку от реализации, прибыль, рентабельность) отображает следующая формула:

$$
\Delta \mathrm{R}=\sum_{i=1}^{m} k a_{i}+\sum_{j=1}^{n} k b_{j}+\sum_{i=1}^{m} \sum_{j=1}^{n} k c_{i j}
$$

где $\mathrm{a}_{\mathrm{i}}-$ изменение результативного показателя деятельности предприятия за счет i-го фактора макросреды;

$\mathrm{b}_{\mathrm{j}}$ - изменение результативного показателя деятельности предприятия за счет $\mathrm{j}$ го фактора внешней макросреды;

$\mathrm{c}_{\mathrm{ij}}$ - изменение результативного показателя деятельности предприятия за счет опосредствованного влияния i-того фактора макросреды через ј-ый фактор внешней среды.

Адекватность полученных результатов зависит не только от достоверности источника, но и от выборочных данных, их правильной систематизации и интерпретации. Другими словами, важно и правильно собрать, и растолковать информацию. С этой целью нужно сформировать иерархический комплекс показателей. Критериями выбора показателей выступят наиболее полное отражение внешней среды и возможность количественной оценки, которая разрешит моделировать конкретное действие факторов риска. Такая модель будет специфической для каждого предприятия. Она должна учитывать возможные виды риска в зависимости от его роли в логистической цепи. Приведенные соображения естественно подводят к выводу о том, что одним из положительных следствий логистизации есть защита предприятия от экономического риска. На самом деле, логистизация практически сводит к минимуму технологический и сырьевой риски, а также уменьшает качественный, ценовой и прибыльный риски. Это происходит за счет согласования действий отдельных предприятий, которые становятся участниками единого процесса движения товара, т.е. имеет место появление синергетического эффекта, присущего всем системам вообще и логистическим 
системам в частности. В логистической цепи такой эффект приобретает характер коммуникационного, такого, что сводит к минимуму риски потерь при перемещении материальных потоков и искажений в информационных потоках. Потребительский и конкурентный риски уменьшаются за счет получения дополнительной информации о субъектах внешней макросреды. Хотя в целом информационный фактор положительно влияет на все виды риска. Снижение экономического риска неизбежно ведет к повышению финансовой стойкости предприятия. Снижение риска в сферах производства и сбыта положительно отображается на объеме продаж и поступлении денежных средств. Другими словами, предприятие получает дополнительную экономию от предупреждения убытка:

$$
\Delta E=\Lambda p_{11} k_{11}+\Delta p_{12} k_{12} \underline{k}_{21}+\Delta p_{22} \boldsymbol{\epsilon}_{22}+k_{23} \underline{\bar{k}}_{31} U_{\max }
$$

где $\Delta \mathrm{E}$ - дополнительная экономия за счет снижения экономического риска;

$\Delta \mathrm{p}_{11}$ - уменьшение вероятности технологического риска;

$\Delta \mathrm{p}_{12}$ - уменьшение вероятности сырьевого риска;

$\mathrm{k}_{11}$ и $\mathrm{k}_{12}$ - коэффициенты, которые определяют влияние на производственный риск соответственно технологического и сырьевого факторов, причем $\mathrm{k}_{11}+\mathrm{k}_{12}=1$;

$\mathrm{k}_{21}, \mathrm{k}_{22}, \mathrm{k}_{23}$ - коэффициенты, которые определяют влияние соответственно качественного и ценового $\left(\mathrm{k}_{21}\right)$, потребительского $\left(\mathrm{k}_{22}\right)$ и конкурентного факторов $\left(\mathrm{k}_{23}\right)$ на сбытовой, причем $\mathrm{k}_{21}+\mathrm{k}_{22}+\mathrm{k}_{23}=1$;

$\Delta \mathrm{p}_{22}$ - уменьшение вероятности сбытового риска за счет потребительского и конкурентного факторов;

$\mathrm{K}_{31}$ - коэффициент, который определяет влияние сбытового фактора на финансовый;

$\mathrm{U}_{\max }$ - максимально возможная величина финансового убытка предприятия.

В этой формуле отображается последовательное влияние блоков рисков согласно стадиям движения оборотных средств. Внутри блока каждый вид риска рассматривается как отдельный элемент. В практических расчетах необходимо делать поправку на возможное внутриблочное влияние рисков. Кроме того, в связи с частым несовпадением периодов действия факторов риска, нужно учитывать прекращение или пролонгацию риска. Характеризуя категорию экономического убытка, можно назвать его количественной оценкой экономического риска. В то же время сам экономический риск выступает фактором экономической безопасности предприятия. Количественно определить эту категорию непросто через субъективность оценки и отсутствие соответствующего формального аппарата (по обыкновению ограничиваются качественной шкалой типа “критическая”, “низкая”, “удовлетворительная”, “высокая”). Кроме того, экономическая безопасность - это динамическая категория, поэтому она требует постоянного мониторинга микро-, и макросреды. Этапы обеспечения экономической безопасности можно представить в виде следующих блоков:

1. Информационный. Сбор данных о потенциальных внутренних и внешних угрозах предприятию.

2. Аналитический. Изучение и обобщение полученной информации. 
3. Блок поиска и разработки. Поиск мероприятий, направленных на сохранение экономической безопасности.

4. Действенный. Реализация предлагаемых мероприятий.

В подтверждение блока 1 заметим, что уровень экономической безопасности зависит как от внутренних изменений, так и от колебаний внешней среды. Таким образом, выделим причины, которые определяют угрозу предприятию: 1) “срыв” одной из подсистем предприятия. В большинстве случаев препятствия одолеваются собственными силами, хотя иногда нужна помощь посторонних консультантов; 2) предприятие не успевает адаптироваться к изменениям внешней среды. Чтобы избежать этого, необходимо прогнозировать ситуацию. Кроме того, нужно четко следить за возможными изменениями, определив ключевые параметры. В случае одновременного наступления рассмотренных событий, вероятность “краха" предприятия приближается к единице. Особенно это касается небольших предприятий, которые не имеют необходимой устойчивости. Поэтому одной из тенденций современной экономики выступает глобализация. Ряд специалистов считает, что в основе процессов глобализации лежат изменения, которые происходят в технико-технологическом, транспортно-комуникационном и информационном базисах экономики. Указанные составляющие снова приводят к логистике, но уже на новом уровне. Применение логистики перемещается из уровня локальных транспортно-складских объединений и сейчас приобретает как региональный, так и национальный масштаб. Причем речь идет не только об удлинении логистической цепи, но и о его расширении, преобразовании на логистическую сеть. Другими словами, происходит разветвление и укрупнение логистического объединения, основанное на углублении специализации. Например, посредник, который обеспечивает продвижение товара к потребителю, передает функцию непосредственной транспортировки транспортно-экспедиционной организации, а последняя уже заключает договор с перевозчиками.

Процесс интеграции затронул и самых производителей продукции и услуг. Логистизация открывает пространство для объединения предприятий с различной рыночной позицией, в основе которого лежит не технологическая общность, а желание сгладить спады, присущиу жизненному циклу любого предприятия.

В зависимости от того, какую стратегию выбирает предприятие и какие методы конкурентной борьбы использует для выживания в рыночных условиях, в логистизации оно находит решение разных задач. Такими задачами являются: расширение сбытовой сети, разработка неординарных видов сервиса, умение оперативно получить необходимые ресурсы, поиск наиболее совершенных материальных ресурсов, которые отвечают новейшим технологиям. Для всех предприятий решаются проблемы, связанные с транспортом, складированием, информационным обеспечением. Кроме того, за счет инвариантности развития снижается потенциальный экономический риск. Таким образом, вхождение в логистическое объединение экономически выгодно предприятию любой рыночной позиции, поскольку приводит к получению положительного эффекта, который образовывается за счет: 1) рационального распределения и потребления материальных ресурсов; 2) полного использования основных фондов; 3) концентрации значительных денежных средств, что является важным фактором экономической безопасности. 
Логистизация допускает объединение предприятий в экономическом, технологическом, организационном и информационном аспектах [5]. Каждый из названных аспектов делает свой вклад в экономическую безопасность (табл. 1).

Таблица 1. Роль логистики в обеспечении экономической безопасности предприятия

\begin{tabular}{|c|l|}
\hline $\begin{array}{c}\text { Аспекты } \\
\text { логистизации }\end{array}$ & \multicolumn{1}{|c|}{ Роль в обеспечении экономической безопасности } \\
\hline Экономический & $\begin{array}{l}\text { Повышение финансовой стойкости за счет средств, которые } \\
\text { раньше тратились на конкурентную борьбу между участниками } \\
\text { процесса движения товара; } \\
\text { Уменьшение финансовых потерь в виде штрафов в связи с } \\
\text { переходом отношений между предприятиям на новый уровень, } \\
\text { что является сотрудничеством участников единого логистического } \\
\text { процесса }\end{array}$ \\
\hline Технологический & Патентная защита новых технологий \\
\hline Организационный & Физическая защита материально-технической базы и персонала \\
\hline Информационный & Разработка программ защиты информации \\
\hline
\end{tabular}

С точки зрения экономической безопасности применение логистики выполняет две функции: 1) страховую, направленную на снижение экономического риска за счет объединения предприятий; 2) развивающую, нацеленную на более полное и эффективное использования ресурсов. При этом не следует забывать, что постановка задачи минимизации расходов возможна лишь при полном удовлетворении нужд потребителей. На качественное обслуживание, в первую очередь, и ориентирована логистическая стратегия (табл. 2).

Таблица 2. Сравнение обычной и логистической стратегий предприятия

\begin{tabular}{|l|l|}
\hline \multicolumn{1}{|c|}{ Традиционная стратегия } & \multicolumn{1}{|c|}{ Логистическая стратегия } \\
\hline $\begin{array}{l}\text { Нормирование запасов по каждому виду } \\
\text { оборотных фондов }\end{array}$ & $\begin{array}{l}\text { Оптимальное распределение запасов между } \\
\text { звеньями логистической цепи }\end{array}$ \\
\hline Выбор транспорта с низкими тарифами & Транспортная консолидация \\
\hline Закупка дешевых ресурсов & $\begin{array}{l}\text { Приобретение ресурсов высокого качества по } \\
\text { принятой цене }\end{array}$ \\
\hline $\begin{array}{l}\text { Устранение отрицательных следствий } \\
\text { производственно-хозяйственной деятельности }\end{array}$ & Превентивное устранение проблем \\
\hline
\end{tabular}

Предприятие производитель может самостоятельно предоставлять услуги (для этого чаще выделяется соответствующий подразделение), а может передать часть полномочий соответствующей организации. В рамках логистической цепи последнее удобнее, поскольку способствует распределению логистических операций с материальными и сервисными потоками (табл. 3). Специализация логистических звеньев делает их эластичными, что приводит к общей стойкости логистического альянса в условиях постоянно меняющихся требований рынка. 
Таблица 3. Сравнение логистических операций с материальными и сервисными потоками

\begin{tabular}{|c|c|c|}
\hline \multirow[b]{2}{*}{ Предмет операции } & \multicolumn{2}{|c|}{ Логистические операции с потоками } \\
\hline & $\begin{array}{c}\text { товарно-материальных } \\
\text { ценностей }\end{array}$ & услуг \\
\hline $\begin{array}{l}\text { Вывод контрактов, которые } \\
\text { касаются основных ресурсов }\end{array}$ & $\begin{array}{l}\text { С поставщиками } \\
\text { (производителями) }\end{array}$ & С нанимаемым персоналом \\
\hline Оформление заказа & $\begin{array}{l}\text { С юридическим лицом на } \\
\text { продолжительный срок }\end{array}$ & $\begin{array}{l}\text { С физический лицом на } \\
\text { короткий срок }\end{array}$ \\
\hline Использование мощностей & $\begin{array}{l}\text { Планирование ассортиментной } \\
\text { загрузки производства }\end{array}$ & $\begin{array}{l}\text { Установление режима работы } \\
\text { предприятия (подраздела) } \\
\text { сервиса }\end{array}$ \\
\hline $\begin{array}{l}\text { Продвижение товара к } \\
\text { потребителю }\end{array}$ & $\begin{array}{l}\text { Выбор оптимальных каналов } \\
\text { движения товара }\end{array}$ & $\begin{array}{l}\text { Определение форм и видов } \\
\text { обслуживания }\end{array}$ \\
\hline $\begin{array}{l}\text { Регламентация } \\
\text { технологического процесса }\end{array}$ & $\begin{array}{l}\text { Выбор системы управления } \\
\text { запасами }\end{array}$ & $\begin{array}{l}\text { Упорядочение потоков } \\
\text { клиентов }\end{array}$ \\
\hline
\end{tabular}

В то же время производитель может не заботиться о качестве услуг (иногда плохое обслуживание ассоциируется с некачественной продукцией), поскольку работает вместе с сервисной фирмой. Наоборот, гибкость логистического сервиса рассматривается как компенсатор рисков при реализации продукции, для которой нельзя гарантировать устойчивый оптимальный режим эксплуатации. Как известно, процесс логистического обслуживания проходит через три стадии: предпродажное обслуживание, предоставление услуг во время продажи, послепродажное обслуживание. На первый взгляд, наиболее важной является первая стадия. Тем не менее, на самом деле, качественное выполнение услуг на второй-третьей стадиях обеспечивает не только повторное обращение реальных потребителей, но и предрасполагает людей, которые каким-нибудь образом общаются с ними потенциальных клиентов. Высокий уровень логистического обслуживания часто определяет величину объема продаж, который прямо влияет на экономическую безопасность всех предприятий - участников логистического объединения. Логистические объединения оказываются более стабильными, чем отдельные предприятия, поскольку являются более сложными и сильными системами (чем выше уровень системы, тем выше ее живучесть). Тем не менее при создании сложных систем могут возникнуть разногласия между вектор-направленностью деятельности ее отдельных участков. Особенно сильно это сказывается в случае преобладания непроизводительных и антиобщественных источников доходов того или иного предприятия [2].

Вхождение Украины в мировое экономическое сообщество повышает значимость логистических объединений. Формирование логистических альянсов приводит к новым взаимоотношениям между предприятиями: переход от конкуренции к кооперации, от тактического к стратегическому планированию общих действий, от краткосрочных к долгосрочным договорам. Необходимость юридического оформления новой организационной структуры требует создания нормативно-правовой базы, где будут представлены положения, которые касаются налогообложения, порядка распределения прибыли, стимулирования инноваций. Законодательная основа даст логистическим объединением импульс к дальнейшему развитию, станет ориентиром при разработке экономической политики. 


\section{3. ВЫВОДЫ}

1. Экономический риск на предприятии можно представить в виде блочной системы. Применение логистики влияет на каждый блок.

2. Современный уровень внедрения логистики на предприятиях определяет новую форму объединения - логистическую сеть, которая предоставляет возможность внутренней интеграции каждого блока логистической цепи.

3. Логистический сервис, который выступает как определяющий фактор объема продаж может выступать как гарант экономической безопасности предприятия.

\section{ЛИТЕРАТУРА}

[1] Ильяшенко С.Н., Составляющие экономической безопасности предприятия и подходы к их оценке , „Актуальные проблемы экономики” 2003, № 3(21), с. 12-19.

[2] Карнаухов С., Синергетика макрологистических систем, „РИСК” 2003, № 2(362), с. 415.

[3] Козаченко Г.В., Пономарьов В.П., Ляшенко О.М., Экономическая безопасность предприятия: сущность и механизм обеспечения: Монография, Изд. Либра, Киев 2003, $280 \mathrm{c}$.

[4] Кузенко Т.Б., Концептуальные подходы к планированию экономической безопасности предприятия // Национализация и приватизация: прошлое, настоящее, будущее. Вестник Харьковского национального университета им. В.Н. Каразина Экономическая серия. - Вып. № 613., Изд. ХНУ им. В.Н.Каразина, Харьков 2003, с. 51-60.

[5] Семенко А.И., Сергеев В.И., Логистика. Основы теории, Изд. „Союз”, Санкт-Петербург 2006, $544 \mathrm{c}$

[6] Часов Ю.Ф., Экономическая безопасность - „всему глава”, „ОКА” 2002, № 10, с. 3-16.

\section{LOGISTIC APPROACH AS FACTOR OF ECONOMIC SECURITY OF ENTERPRISE}

In the article the application of logistics as way to achieve economic security and stability of an enterprise is examined. Concepts and types of risk as to the economic category are described. The optimal form of logistics association, adequate to the modern economy is featured. Logistics service in the aspect of economic security of enterprise is considered.

DOI: $10.7862 /$ rz.2012.zim.20 